\title{
The Longitudinal Effect of Vertigo and Dizziness Symptoms on Psychological Distress
}

\section{Symptom-Related Fears and Beliefs as Mediators}

\author{
Katharina Radziej, Dipl.-Psych., *† Thomas Probst, Dr. rer. nat., $\neq \mathcal{f}$ \\ Karina Limburg, MSc, *广 Andreas Dinkel, Dr. rer. nat., * \\ Marianne Dieterich, Univ. Prof. Dr. med., $\dagger$ // and Claas Lahmann, Univ. Prof. Dr. med. 9
}

\begin{abstract}
Despite the frequent observation that vertigo and dizziness (VD) disorders may trigger or exacerbate secondary psychiatric comorbidities, there is limited understanding of the mechanisms underlying this development. To address this gap, we investigated whether symptom-related fears and cognitions as indicated by questionnaire-based measures are mediators of the longitudinal effect of VD symptoms on anxiety and depression after 1 year. We analyzed data from a large study with patients of a treatment center specialized in vertigo $(N=210)$. Simple and multiple parallel mediation models strengthened our hypothesis that fear of bodily sensations and cognitions about these symptoms play a mediating role in the relationship between VD symptoms and psychopathology at follow-up after baseline scores of the outcome were controlled for. Results are discussed within a cognitive theory framework and point to the potential benefits of interventions that modify symptom-related beliefs and fears via cognitive psychotherapy in this therapeutically underserved population.
\end{abstract}

Key Words: Dizziness, vertigo, somatoform disorder, somatic symptom disorder, symptom beliefs

(J Nerv Ment Dis 2018;206: 277-285)

$\mathrm{Ve}_{\mathrm{tin}}$ ertigo and dizziness (VD) rank among the most common symptoms in adults with a lifetime prevalence of approximately $30 \%$ (Neuhauser, 2007; Tusa, 2009). The underlying causes of those symptoms vary largely, ranging from peripheral and central vestibular disorders or other organic causes to somatoform disorders and all combinations thereof. Irrespective of their etiology, VD symptoms are often experienced as severely distressing. They are associated with handicap in daily and working lives (Lahmann et al., 2015; Yardley et al., 1992) as well as a significant reduction of both mental and physical healthrelated quality of life (Weidt et al., 2014). Subjective impairment and health care use due to their VD are further increased if patients are characterized by psychiatric comorbidity (Lahmann et al., 2015; Wiltink et al., 2009). Not only do they show a higher persistency of symptoms (Best et al., 2009b), but psychological distress also has been shown to

*Department of Psychosomatic Medicine and Psychotherapy, Klinikum rechts der Isar, Technical University of Munich; †German Centre for Vertigo and Balance Disorders, Klinikum Großhadern, Ludwig-Maximilians-Universitaet Muenchen, Munich; †Department of Clinical Psychology and Psychotherapy, Georg-AugustUniversitaet Goettingen, Goettingen, Germany; §Department of Psychotherapy and Bio-Psycho-Social Health, Danube University Krems, Krems, Austria; ||Department of Neurology, Ludwig-Maximilians-Universitaet Muenchen, Munich; and Department of Psychosomatic Medicine and Psychotherapy, Faculty of Medicine, University of Freiburg, Freiburg, Germany.

Ethical approval: The ethics committee of the Medical Department of the Ludwig-Maximilians-Universitaet, Munich, Germany, reviewed and approved this study (ref: 108-10).

Send reprint requests to Katharina Radziej, Dipl.-Psych., Klinik und Poliklinik für Psychosomatische Medizin und Psychotherapie, Klinikum rechts der Isar,

Technische Universität München, Langerstr. 3/I, D-81675 München, Germany. E-mail: k.radziej@tum.de.

Copyright (C) 2018 Wolters Kluwer Health, Inc. All rights reserved.

ISSN: 0022-3018/18/20604-0277

DOI: $10.1097 /$ NMD.0000000000000791 be important in the process how VD symptoms lead to VD handicap (Probst et al., 2017). More often than not VD symptoms have been linked to such psychiatric comorbidity, regardless of whether studies were conducted from the vantage point of vestibular deficits or psychopathology (Best et al., 2009a; Bigelow et al., 2015; Staab and Ruckenstein, 2003). The highest rates of psychiatric comorbidity have been reported for vestibular migraine, Ménière's disease, vestibular paroxysmia, and functional VD (Eckhardt-Henn et al., 2008; Lahmann et al., 2015). Furman et al. (2005) suggested neuroanatomical connections between vestibular stimuli and the emotional response processing system to explain the comorbidity of VD and psychiatric disorders. However, cross-sectional studies found that emotional distress was independent of the amount of vestibular deficit (Best et al., 2006; Pérez et al., 2003). The extent of vestibular dysfunction also had no impact over the course of time on the development of secondary psychiatric disorders (Best et al., 2009a; Eagger et al., 1992). On that account, the study at hand focuses on the question as to what does act as the intermediary between VD symptoms and the development or exacerbation of psychological distress.

Cognitive theory suggests that dysfunctional cognitions contribute to psychological distress in a causal manner (e.g., Beck, 2005), an association that was corroborated in a recent meta-analysis (Vîslă et al., 2016). With regard to VD, patients frequently present with individual patterns of thoughts and feelings related to their VD symptoms that fulfil the B criterion of the Diagnostic and Statistical Manual of Mental Disorders, 5th Edition, somatic symptom disorder (Limburg et al., 2016); these may include disproportionate thoughts about the symptoms' seriousness, a persisting high level of anxiety about health or symptoms, and excessive time and energy devoted to these symptoms or health concerns (American Psychiatric Association [APA], 2013). Cognition has been described as a potent modulator of somatic and psychological symptoms via processes such as somatosensory amplification (Barsky, 1992). This concept describes an altered perception of somatic stimuli that includes a heightened attentional focus on bodily sensations and a tendency to appraise them as intense, alarming, and signs of a threatening disease. Somatosensory amplification reflects aspects of cognitive processing rather than mere interoceptive sensitivity; it has been proposed to explain some of the variability in somatic symptomatology found among different patients with the same medical disorder and one factor mediating the link between somatic and psychological symptoms (Nakao and Barsky, 2007).

Recent theoretical models have taken a closer look at the interplay between interoceptive and self-referential cognitive processes with regard to anxiety and depression and suggested that "external cues or internal thought processes might generate an anticipation of aversive body states that $[\ldots]$ [act] as a motivating signal for individuals to withdraw (depression) or avoid (anxiety)." (Paulus and Stein, 2010, p 456). That is, if bodily symptoms such as VD are experienced as particularly troubling, potentially uncontrollable, or persistent, this appraisal guides action planning and may thereby contribute to a negative self-view, for 
example, as being helpless and less self-efficient (depression) or an increased attentional bias toward indicators of potential threat associated with avoidance behavior (anxiety). With regard to catastrophic thinking of this sort (i.e., the tendency to overinterpret the likelihood or intensity of potential negative consequences of symptoms, misinterpretation of somatic sensations as signs of a serious illness, or ruminating about worst-case outcomes), there is extensive literature on its correlational and predictive value in a variety of disorders (Gellatly and Beck, 2016). However, only few studies have investigated the role of symptom-related cognitions in patients presenting with VD: in their prospective study on patients with an acute episode of vestibular neuritis, Godemann et al. found catastrophic thinking, persistent fear of vertigo, and panic-related thoughts to predict chronic vertigo (Godemann et al., 2004) and the development of secondary somatoform and panic disorder (Godemann et al., 2006). Another study demonstrated that for the development of secondary somatoform dizziness after a peripheral vestibular disorder, the fear of bodily sensations was relevant in the interaction with the initial severity of dizziness (Heinrichs et al., 2007). In Ménière's disease, maladaptive beliefs about the consequences of VD even appeared to be more disabling than symptoms themselves (Yardley et al., 2001).

Although the aforementioned studies mainly focused on the role of symptom-related fears and beliefs in the development or perpetuation of (somatoform) VD symptoms, there is, to our knowledge, a lack of investigations on how fears and cognitions contribute to the effect VD symptoms longitudinally exert on the development of anxiety and depression. Previous studies on this issue have almost exclusively focused on patients with Ménière's disease. Illness uncertainty/ unpredictability and illness intrusiveness have been discussed as factors contributing to depression in these patients (Arroll et al., 2012). With regard to anxiety, Kirby and Yardley (2009) found that vertigo can lead to increased anxiety, if patients with Ménière's disease reported high intolerance of uncertainty and less understanding of their illness.

The scarcity of investigations in that area is of particular concern because current psychotherapeutic efforts may be effective in reducing VD symptoms but mostly failed to achieve clinically relevant improvements concerning the frequently associated symptoms of anxiety or depression (Schmid et al., 2011).

Aim of the study. The current study seeks to explore whether the longitudinal effect of VD symptoms (assessed at baseline) on anxiety and depression (assessed at 12-month follow-up) is mediated by symptomrelated fears and cognitions (assessed at the 6-month follow-up). We therefore reanalyzed data from a large study with patients of a treatment center specialized in vertigo covering a variety of VD disorders (Lahmann et al., 2012).

Hypothesis. We hypothesized that fear of bodily sensations and cognitions about these symptoms would longitudinally mediate the effect initial VD symptoms have on the development of anxiety and depression.

\section{METHODS}

\section{Study Design and Sample Characteristics}

This longitudinal study encompasses three assessment points: baseline (t0), first follow-up after 6 months ( $\mathrm{t} 1)$, and second follow-up after 12 months ( $\mathrm{t} 2$ ). Patients were recruited via routine care appointments at the German Centre for Vertigo and Balance Disorders at the University Hospital Munich, Campus Großhadern, between May 2010 and June 2012. All patients had been referred with dizziness or imbalance as their primary complaint. Patients were excluded if they were less than 18 years of age, had no sufficient German language skills, or had a neurodegenerative disorder (e.g., dementia). A total of $686(\approx 80 \%)$ of 860 eligible patients gave their consent to participate in the study. There were 350 and 360 patients who took part in the first and second followup assessment, respectively.
For this study, only those patients for which scores on the following questionnaires could be computed were analyzed: Vertigo Symptom Scale (VSS; vertigo subscale) at t0, Body Sensations Questionnaire (BSQ), and Agoraphobic Cognitions Questionnaire (ACQ) at t1, Beck Depression Inventory (BDI-II), and Beck Anxiety Inventory (BAI) at t2. A total of 210 patients fulfilled this inclusion criterion. Patients that were excluded from the analyses did not differ from included patients with regard to sex distribution or any of the questionnaire-based measures at baseline. However, excluded patients were significantly younger (included: mean $=57.4$ yrs $[\mathrm{SD}=15.6]$; excluded: mean $=54.4 \mathrm{yrs}$ $[\mathrm{SD}=16.3] ; t[685]=2.30, p=0.022$, Hedges' $g=0.18$ ).

This study was part of the Munich Diagnostic and Predictor Study of Somatoform Dizziness, which is described in detail elsewhere (Lahmann et al., 2012). All participants gave written informed consent, and the study was approved by the ethics committee of the University of Munich.

\section{Diagnostic Assessment and Patient Self-Report Questionnaires}

All patients underwent structured collection of their histories and a systematic and standardized physical examination, including complete neurological, neurootological, and neuroophthalmological examination. Vestibular testing included the head impulse test, measurements of subjective visual vertical and ocular torsion, and video-oculography with caloric irrigation. Frequencies of respective diagnoses are listed in the Results section. The following self-report questionnaires were administered to patients:

(1) VD symptoms. VD symptoms were assessed using the VSS (Yardley et al., 1992). The VSS (German version by Tschan et al., 2008) consists of two scales measuring vertigo-balance symptoms (VSS-VER; e.g., dizziness, unsteadiness, and light-headedness) and vertigo-induced autonomic-anxiety symptoms (VSS-AA; e.g., sweating, pounding heart, and breathing difficulties). In the present study, only the VSS-VER score was analyzed. It comprises 19 items scored on a 5-point Likert scale ranging from 0 (never) to 4 (more than once a week). The VSS shows good test-retest reliability and construct validity (Yardley et al., 1992; Tschan et al., 2008). For the 210 patients of the present study, the Cronbach's alpha of the VSS-VER scale at t0 was $\alpha=0.86$.

(2) Anxiety and depression. Psychological distress, namely, symptoms of anxiety and depressive disorders, was assessed using the BAI (German version by Margraf and Ehlers, 2007) and the revised BDI-II (German version by Hautzinger et al., 2006). The BDI-II assesses depression severity during the past 2 weeks; 21 Items are rated on a 4-point scale (0-3). It is a psychometrically sound instrument (Kühner et al., 2007) with a Cronbach's alpha of $\alpha=0.92$ at t 2 in our sample. The BAI is composed of 21 items measuring severity of anxiety symptoms during the last 7 days. Items are scored on a 4-point Likert scale ranging from 0 (not at all) to 3 (strongly). The inventory shows good reliability and validity (Margraf and Ehlers, 2007). The BAI at t 2 reached a Cronbach's alpha of $\alpha=0.93$ in our sample.

(3) Cognitions and fears with regard to bodily symptoms. To assess the fear of physical symptoms and panic-related cognitions, the BSQ and the ACQ out of a questionnaire series by Chambless et al. (1984) were applied in their German version (Ehlers and Margraf, 2001). The BSQ is composed of 17 items measuring the intensity of fear with respect to body sensations (e.g., vertigo, palpitations, and feeling of not being able to breathe). On a 5-point Likert scale, patients indicate to which extent they worry about a particular symptom, ranging from 1 (not at all anxious) to 5 (extremely anxious). The ACQ is a 14-item questionnaire designed to measure maladaptive thoughts about the potential for catastrophic consequences arising from anxiety or panic. Thoughts such as "I will have a heart attack" or "I will not be able to control myself" are rated on a 5-point Likert scale (1-the thought never occurs to me; 5 - the thought always occurs to me). Two subscales can be 
computed: "physical crisis" (five items) and "loss of control" (seven items). Overall, the German versions of the BSQ and ACQ scales showed good internal consistency and reliability. The Cronbach's alphas of both questionnaires were high in our sample (BSQ at t1: $\alpha=0.93$; ACQ at t1: $\alpha=0.81$ ). The ACQ subscales had acceptable reliability (ACQ physical crisis at t1: $\alpha=0.72$; ACQ loss of control at t1: $\alpha=0.76)$.

\section{Statistical Analyses}

All statistical analyses were performed using SPSS 22 statistical package. To test for differences among included versus excluded patients with regard to sex, age, and self-report questionnaire scores, categorical variables were analyzed using the chi-square tests, and continuous baseline variables were analyzed using independent samples $t$-tests. Pearson correlation coefficients were calculated to assess the correlations between the measures analyzed in the study; Bonferroni correction was applied to correct for multiple testing.

To evaluate the mediating role of symptom-related fears and cognitions (BSQ, ACQ, and its subscales) at $\mathrm{t} 1$ in the relationship between VD symptoms (VSS-VER) at t0 and psychological distress (BDI-II, BAI) at 2 , model 4 within PROCESS (Hayes, 2013), a macro for SPSS for moderation and mediation analyses, was used. PROCESS uses bootstrapping to estimate confidence intervals (CIs) of indirect effects (IEs). For all mediation analyses in the present study, the bias-corrected bootstrap CI method was selected with 10.000 bootstrap samples. To correct for multiple testing, the confidence level for CI was set to $98.75 \%$, taking into account that four mediation models were calculated for each outcome measure. Completely standardised indirect effects (CS) (Preacher and Kelley, 2011) were computed as a measure of effect size and considered small if $0.01 \leq \mathrm{CS}<0.09$, medium if $0.09 \leq \mathrm{CS}<0.25$, and large if $\mathrm{CS} \geq 0.25$. The $p$ value was set to $p<0.05$ in all analyses. Before analyses, bivariate scatterplots of the antecedent, mediator, and consequent variables were examined to evaluate the assumption of linear relations. To test for multicollinearity, variance inflation factors (VIFs) were computed for every step in the mediation analyses; with all VIF less than 1.6 , it is unlikely that this might have introduced substantial error to coefficient estimates of individual predictors.

Because the subsample the analyses are based on differed from the excluded part of the sample in terms of age, it was included as a covariate across mediation analyses. To control for baseline values of the outcome measures, the BAI and BDI-II scores at the 12-month follow-up were entered into separate univariate simple linear regression analyses with their baseline scores serving as the independent variable, respectively. Residuals from these analyses were used as outcome variable in all further mediation models.

Two simple mediation models and two multiple parallel mediation models were calculated for each of the two outcome variables (BDI-II and BAI at $\mathrm{t} 2$ ). Diagrams of the mediation models are illustrated in Figure 1. In all mediation models, the VSS vertigo score at t0 was entered as the independent variable.

First, simple mediation models evaluated symptom-related fears (BSQ; model [A]) and symptom-related cognitions (ACQ; model [B]), separately, as mediators of the effect that VD symptoms at baseline exert on the respective outcome variable. Second, both ACQ and BSQ were included in a multiple parallel mediation model $[C]$ to evaluate whether both measures remain significant mediators when statistically controlling for the other one. Finally, model [D] contrasted the two ACQ subscales in a multiple parallel mediation model to explore whether one of the two aspects of symptom-related cognitions outweighs the other in mediating the relationship between VD symptoms and the outcome measures. We note that model [D] was exploratory in nature and did not correspond directly to the study hypothesis.

\section{RESULTS}

\section{Characteristics and Diagnoses of the Study Sample}

Of the 210 analyzed patients, 119 (57\%) were female. The most frequent diagnoses were functional VD $(n=71)$, benign paroxysmal positional vertigo $(n=37)$, Ménière's disease $(n=37)$, and vestibular migraine $(n=31)$. More than one third of patients received more than one diagnosis. Descriptive statistics of all questionnaire-based measures are presented in Table 1 . Across the diagnostic subgroups, $20 \%$ of patients $(n=42)$ showed moderate to severe levels of depression (BDI-II score $\geq 20)$ at the second follow-up, and $32 \%(n=66)$ showed moderate to severe levels of anxiety according to their BAI scores $(\geq 16)$ after 1 year. Table 2 gives an overview of the diagnoses and the respective descriptives of the mediator variables of interest.

\section{Correlations Between the Measures}

Pearson correlation coefficients between all the questionnairebased measures (VSS-VER at $\mathrm{t} 0, \mathrm{ACQ}$ at $\mathrm{t} 1, \mathrm{BSQ}$ at $\mathrm{t} 1, \mathrm{BDI}-\mathrm{II}$ at $\mathrm{t} 0$

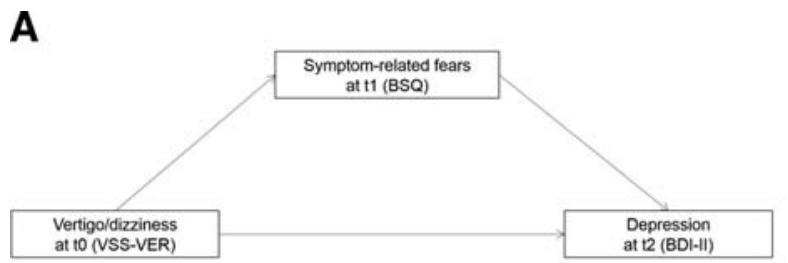

B

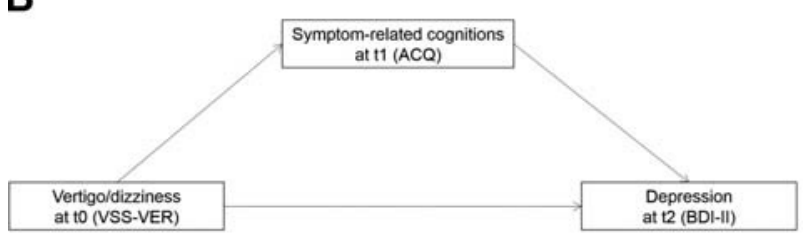

C
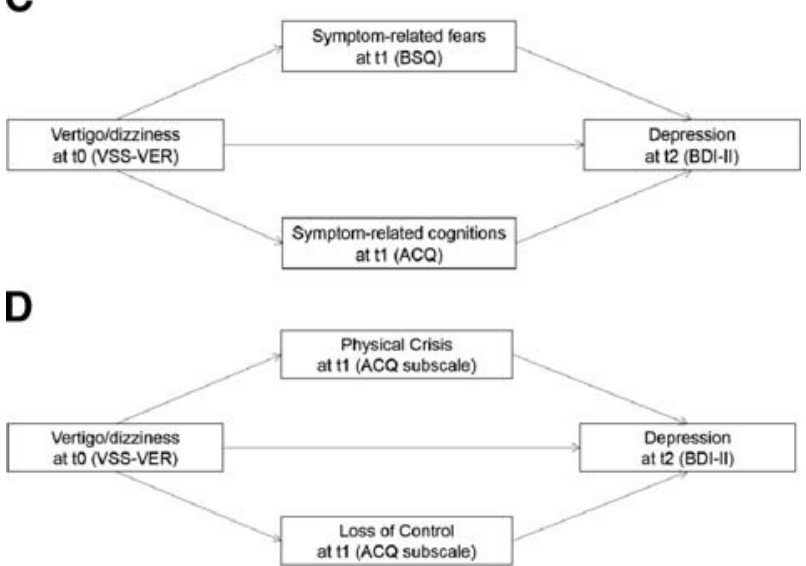

FIGURE 1. Diagrams of mediation models. (A) Simple mediation model investigating symptom-related fears as a mediator between VD symptoms and depression. (B) Simple mediation model investigating symptom-related cognitions as a mediator between VD symptoms and depression. (C) Multiple parallel mediation model investigating both symptom-related fears and cognitions as mediators between VD symptoms and depression. (D) Multiple parallel mediation model investigating physical concerns and loss of control as mediators between VD symptoms and depression. Mediation models with regard to anxiety symptoms as outcome variable (t2) were defined accordingly. 
TABLE 1. Descriptive Statistics of Questionnaire-Based Measures $(N=210)$

\begin{tabular}{lcc}
\hline & $M$ (SD) & Range \\
\hline VD symptoms (t0) & & \\
$\quad$ VSS-VER & $0.96(0.71)$ & $0.00-3.53$ \\
Symptom-related cognitions (t1) & & \\
$\quad$ ACQ & $1.31(.33)$ & $1.00-3.07$ \\
$\quad$ ACQ physical crisis & $1.37(.46)$ & $1.00-3.40$ \\
$\quad$ ACQ loss of control & $1.30(.40)$ & $1.00-3.29$ \\
Symptom-related fears (t1) & & \\
$\quad$ BSQ & $1.88(.66)$ & $0-4.00-41$ \\
Depression (t2) & $11.90(9.47)$ & \\
$\quad$ BDI-II & & $0-56$ \\
Anxiety (t2) & $12.85(11.08)$ & \\
$\quad$ BAI &
\end{tabular}

and $\mathrm{t} 2$, and $\mathrm{BAI}$ at $\mathrm{t} 0$ and $\mathrm{t} 2$ ) are illustrated in Table 3 . Correlations were all positive and statistically significant.

\section{Mediation Analyses Between Vertigo Symptoms and Depression}

Throughout the mediation models, age did not contribute significantly to any of the regression analyses, that is, regression coefficients for age as a covariate in the prediction of the mediator or outcome variables failed to reach statistical significance. The results of the simple mediation models analyzing the IE of vertigo symptoms on depression after 1 year through symptom-related fears and cognitions (models [A] and $[\mathrm{B}]$ ) revealed that both variables of interest at $\mathrm{t} 1$ functioned as significant mediators with the upper and lower levels of the CI of the IE being above zero. With regard to symptom-related fears (BSQ), the $\mathrm{CS}$ amounted to 0.06 , indicating a small effect after baseline BDI-II scores had been netted out $(\mathrm{IE}=0.53$, Boot $\mathrm{SE}=0.25$; Boot $\mathrm{LLCI}=0.07$, Boot ULCI $=1.35$ ). When symptom-related cognitions (ACQ) were taken into account in a simple mediation model, a CS of 0.11 indicated a medium effect $(\mathrm{IE}=0.96$, Boot $\mathrm{SE}=0.32$; Boot $\mathrm{LLCI}=0.32$, Boot ULCI $=1.95)$.

Only ACQ remained a significant mediator when both ACQ and BSQ scores were included in a multiple parallel mediation model (ACQ: $\mathrm{IE}=0.92$, Boot $\mathrm{SE}=0.33$; Boot $\mathrm{LLCI}=0.27$, Boot $\mathrm{ULCI}=1.98$; BSQ: $\mathrm{IE}=0.07$, Boot $\mathrm{SE}=0.22$; Boot $\mathrm{LLCI}=-0.45$, Boot
TABLE 2. ACQ and BSQ Scores by Diagnosis and Number of Diagnoses

\begin{tabular}{lccc}
\hline & & ACQ (t1), & BSQ (t1), \\
Diagnosis & $\boldsymbol{n}(\%)$ & $\boldsymbol{M}(\mathbf{S D})$ & $\boldsymbol{M}(\mathbf{S D})$ \\
\hline Functional VD & $71(34 \%)$ & $1.39(0.400)$ & $2.01(0.524)$ \\
Benign paroxysmal positional vertigo & $37(18 \%)$ & $1.28(0.305)$ & $1.81(0.695)$ \\
Ménière's disease & $37(18 \%)$ & $1.26(0.282)$ & $1.88(0.693)$ \\
Vestibular migraine & $31(15 \%)$ & $1.34(0.301)$ & $1.86(0.649)$ \\
Bilateral vestibulopathy & $24(11 \%)$ & $1.26(0.292)$ & $1.67(0.524)$ \\
Multisensory deficit & $16(8 \%)$ & $1.24(0.197)$ & $1.93(0.719)$ \\
Central vertigo & $15(7 \%)$ & $1.38(0.332)$ & $1.97(0.643)$ \\
Vestibular paroxysmia & $13(6 \%)$ & $1.41(0.330)$ & $2.00(0.617)$ \\
Polyneuropathy & $12(6 \%)$ & $1.26(0.187)$ & $1.91(0.587)$ \\
Vestibular neuritis & $12(6 \%)$ & $1.24(0.302)$ & $1.77(0.583)$ \\
Unilateral vestibular loss & $8(4 \%)$ & $1.34(0.420)$ & $2.02(0.867)$ \\
Not otherwise specified & $26(12 \%)$ & $1.29(0.320)$ & $1.88(0.746)$ \\
No. diagnoses & & & \\
$\quad n=1$ & $135(64 \%)$ & $1.30(0.340)$ & $1.85(0.671)$ \\
$\quad n=2$ & $56(27 \%)$ & $1.33(0.332)$ & $1.85(0.640)$ \\
$n=3$ & $19(9 \%)$ & $1.34(0.278)$ & $2.12(0.624)$ \\
\hline
\end{tabular}

$\mathrm{ULCI}=0.70$; model $[\mathrm{C}]$; see Table 4). The combination of the two variables accounted for a CS of 0.11 (BSQ: $\mathrm{CS}=0.01$; $\mathrm{ACQ}$ : $\mathrm{CS}=0.10$ ) after baseline scores of the outcome had been controlled for. In a pairwise comparison between the specific IEs, body-related cognitions (ACQ) made a significantly larger contribution than symptom-related fears (BSQ; IE contrast $=0.86$, Boot $\mathrm{SE}=0.46$; Boot $\mathrm{LLCI}=0.05$, Boot ULCI $=1.87)$. Finally, when the two ACQ subscales were included in a multiple parallel mediation model (model [D]; see Table 5), their combination fully mediated the effect vertigo symptoms had on depression after baseline depression scores were controlled for, with a $\mathrm{CS}$ of 0.10 indicating a medium effect size. Only for the ACQ subscale loss of control, the CI of the IE did not include zero (ACQ loss of control: $\mathrm{IE}=0.51$, Boot SE $=0.28$; Boot LLCI $=0.03$, Boot $\mathrm{ULCI}=1.47$; although the IE of the ACQ subscale physical crisis was significant on a $5 \%$ alpha level, it failed to reach significance when multiple testing was corrected for (ACQ physical crisis: $\mathrm{IE}=0.41$, Boot $\mathrm{SE}=0.22$; Boot LLCI $=-0.01$, Boot ULCI $=1.15$ ). In a pairwise comparison between the specific IEs, neither of the two ACQ subscales made a significantly larger contribution than the other (IE contrast $=-0.10$, Boot $\mathrm{SE}=0.39$; Boot LLCI $=-1.00$, Boot ULCI $=0.58$ ).

TABLE 3. Pearson Correlation Coefficients Between the Measures Used in This Study $(N=210)$

\begin{tabular}{|c|c|c|c|c|c|c|c|}
\hline & $\begin{array}{l}\text { VD Symptoms } \\
\text { (VSS-VER, t0) }\end{array}$ & $\begin{array}{c}\text { Symptom-Related } \\
\text { Cognitions (ACQ, t1) }\end{array}$ & $\begin{array}{c}\text { Symptom-Related } \\
\text { Fears (BSQ, t1) }\end{array}$ & $\begin{array}{l}\text { Depression } \\
\text { (BDI-II, t2) }\end{array}$ & $\begin{array}{l}\text { Depression } \\
\text { (BDI-II, t0) }\end{array}$ & $\begin{array}{c}\text { Anxiety } \\
\text { (BAI, t2) }\end{array}$ & $\begin{array}{l}\text { Anxiety } \\
\text { (BAI, t0) }\end{array}$ \\
\hline VSS-VER (t0) & - & $.32 *$ & $.27 *$ & $.26^{*}$ & $.31 *$ & $.28 *$ & $.35^{*}$ \\
\hline ACQ (t1) & - & - & $.58^{*}$ & $.62 *$ & $.54^{*}$ & $.56^{*}$ & $.55^{*}$ \\
\hline BSQ (t1) & - & - & - & $.47 *$ & $.44^{*}$ & $.54 *$ & $.51 *$ \\
\hline BDI-II (t2) & - & - & - & - & $.75^{*}$ & $.70^{*}$ & $.58 *$ \\
\hline BDI-II (t0) & - & - & - & - & - & $.57 *$ & $.72 *$ \\
\hline BAI (t2) & - & - & - & - & - & - & $.62 *$ \\
\hline $\mathrm{BAI}(\mathrm{t} 0)$ & - & - & - & - & - & - & - \\
\hline
\end{tabular}

Note: t0 indicates baseline; t1, 6-month follow-up; t2, 12-month follow-up.

* $p<0.0025$ (Bonferroni correction). 
TABLE 4. Symptom-Related Fears (BSQ) and Cognitions (ACQ) as Mediators of the Effect Vertigo Symptoms Exert on Depression (BDI-II) in a Multiple Mediation Model $(N=210)$

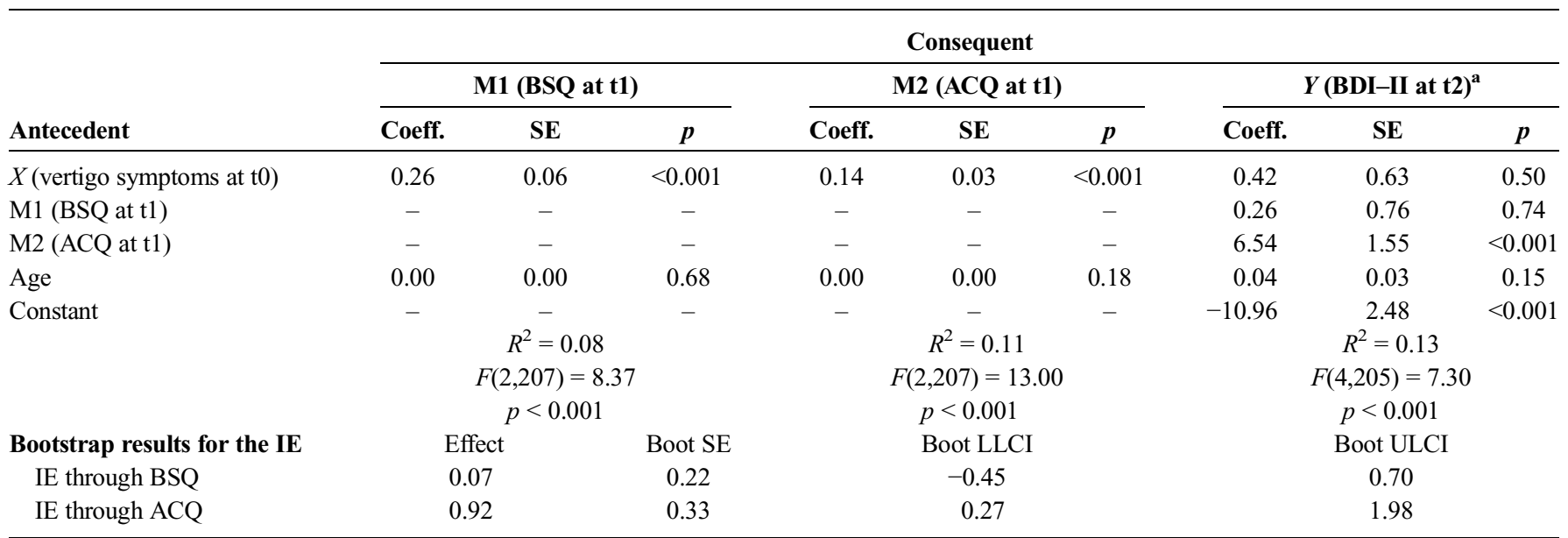

${ }^{a}$ Residuals from simple linear regression analysis were entered into mediation analyses to control for BDI-II baseline scores.

Note: $X$ indicates independent variable; $Y$, outcome variable; M, mediator; Coeff., coefficient; SE, standard error; Boot, bootstrapping; LLCI, lower level of the $98.75 \%$ CI; ULCI, upper level of the $98.75 \%$ CI.

\section{Mediation Analyses Between Vertigo Symptoms and Anxiety}

The results of the simple mediation models analyzing the IE of vertigo symptoms on anxiety after 1 year through symptom-related fears and cognitions (models [A] and [B]) showed that both variables of interest at $\mathrm{t} 1$ functioned as significant mediators. With regard to symptom-related fears (BSQ), the CS amounted to 0.08, indicating a small effect after baseline BAI scores had been controlled for ( $\mathrm{IE}=0.96$, Boot $\mathrm{SE}=0.33$; Boot LLCI $=0.34$, Boot ULCI $=2.09$ ). When symptomrelated cognitions (ACQ) were taken into account in a simple mediation model, a CS of 0.09 indicated a medium effect $(\mathrm{IE}=1.09$, Boot $\mathrm{SE}=0.40$; Boot LLCI $=0.29$, Boot ULCI $=2.40$ ).
Only BSQ remained a significant mediator when both the ACQ and BSQ scores were included in a multiple parallel mediation model (ACQ: IE $=0.73$, Boot $\mathrm{SE}=0.41$; Boot LLCI $=-0.13$, Boot $\mathrm{ULCI}=2.08$; $\mathrm{BSQ}$ : $\mathrm{IE}=0.60$, Boot $\mathrm{SE}=0.29$; Boot $\mathrm{LLCI}=0.03$, Boot $\mathrm{ULCI}=1.56$; model [C]; see Table 6). The combination of the two variables accounted for a CS of 0.11 (BSQ: $\mathrm{CS}=0.05$; $\mathrm{ACQ}$ : $\mathrm{CS}=0.06$ ) after BAI baseline scores had been controlled for. The CI of the contrast of the IEs did not indicate that either of them made a significantly larger contribution (IE contrast $=-0.13$, Boot $\mathrm{SE}=0.57$; Boot $\mathrm{LLCI}=-1.32$, Boot ULCI $=0.94)$.

When the two ACQ subscales were contrasted in a multiple parallel mediation model (model [D]; see Table 7), only the ACQ subscale physical crisis remained a significant mediator (ACQ physical crisis:

TABLE 5. ACQ-Physical Crisis and ACQ Loss of Control as Mediators of the Effect Vertigo Symptoms Exert on Depression (BDI-II) in a Multiple Mediation Model $(N=210)$

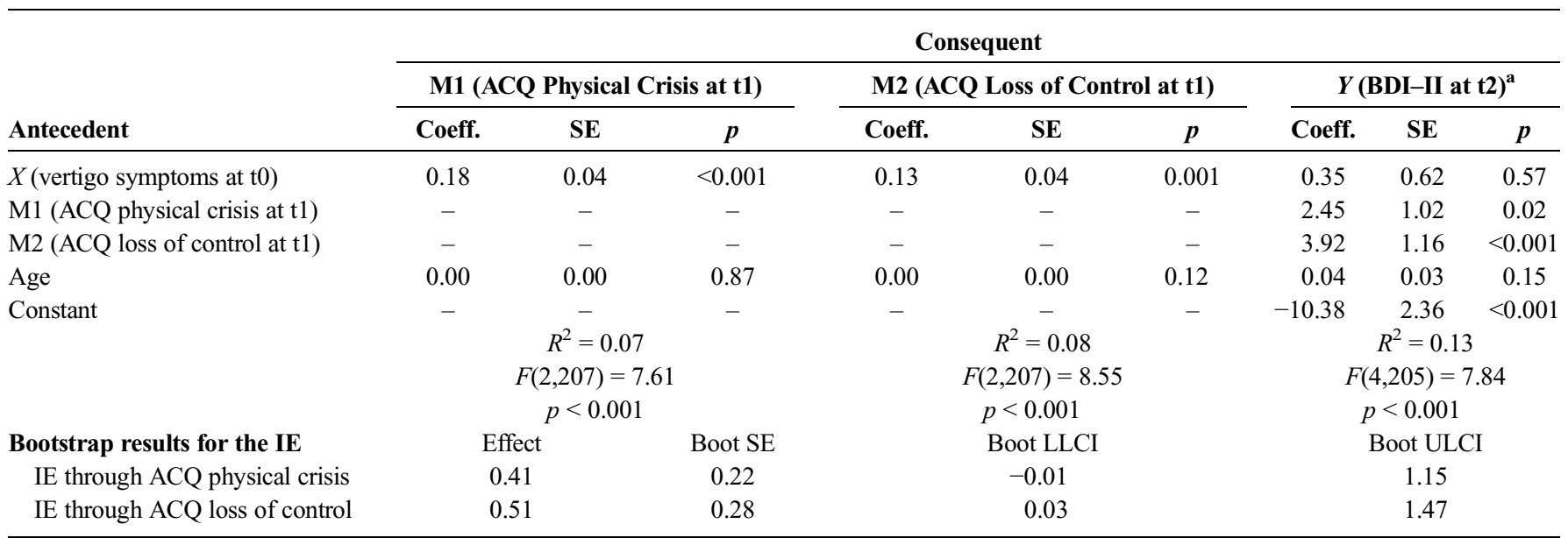

${ }^{\mathrm{a}}$ Residuals from simple linear regression analysis were entered into mediation analyses to control for BDI-II baseline scores.

Note: $X$ indicates independent variable; $Y$, outcome variable; M, mediator; Coeff., coefficient; SE, standard error; Boot, bootstrapping; LLCI, lower level of the $98.75 \%$ CI; ULCI, upper level of the $98.75 \%$ CI 
TABLE 6. Symptom-Related Fears (BSQ) and Cognitions (ACQ) as Mediators of the Effect Vertigo Symptoms Exert on Anxiety (BAI) in a Multiple Mediation Model $(N=210)$

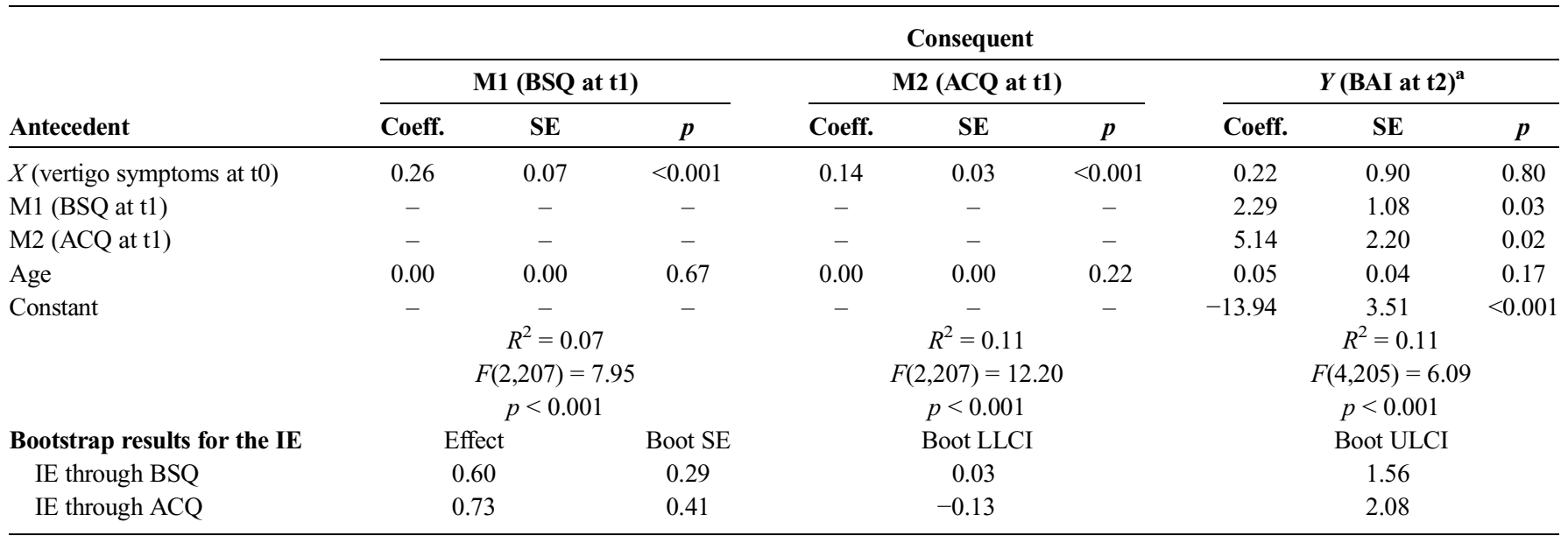

${ }^{a}$ Residuals from simple linear regression analysis were entered into mediation analyses to control for BAI baseline scores.

Note: $X$ indicates independent variable; $Y$, outcome variable; M, mediator; Coeff., coefficient; SE, standard error; Boot, bootstrapping; LLCI, lower level of the $98.75 \%$ CI; ULCI, upper level of the $98.75 \%$ CI.

$\mathrm{IE}=1.01$, Boot $\mathrm{SE}=0.47 ;$ Boot LLCI $=0.21$, Boot $\mathrm{ULCI}=2.60 ; \mathrm{ACQ}$ loss of control: $\mathrm{IE}=0.20$, Boot $\mathrm{SE}=0.26$; Boot $\mathrm{LLCI}=-0.46$, Boot $\mathrm{ULCI}=1.03$ ). Its CS amounted to 0.08 , indicating a small effect. However, the descriptive difference of the IEs of the ACQ subscales did not meet criteria for statistical significance (IE contrast $=0.82$, Boot $\mathrm{SE}=0.60$; Boot LLCI $=-0.12$, Boot $\mathrm{ULCI}=2.31$ ).

\section{DISCUSSION}

In this study, we examined the role of symptom-related fears and cognitions as mediators of the longitudinal effect that VD symptoms exert on symptoms of anxiety and depression after 1 year. The results across all simple mediation models support our hypothesis that their association is (at least partly) mediated by both dysfunctional apprehensions and fear of physical symptoms after baseline levels of depression and anxiety had been netted out. More precisely, reacting to physical symptoms fearfully and with anxiety-related cognitions such as fear of having a crisis or losing control over their body contributed to the process to develop psychological distress after a VD disorder. Overall, effect sizes were small to medium; because total effects were small in the first place, only a limited amount of variance in the outcome variables could be explained with the models at hand.

With regard to depression, maladaptive cognitions about bodily sensations made a significantly larger contribution than symptomrelated fears. In particular, a lack of perceived level of control with regard to symptoms contributed to the development of depression after VD.

With regard to anxiety, body-related fears and cognitions accounted for a similar amount of the total effect that VD had on

TABLE 7. ACQ Physical Crisis and ACQ Loss of Control as Mediators of the Effect Vertigo Symptoms Exert on Anxiety (BAI) in a Multiple Mediation Model $(N=210)$

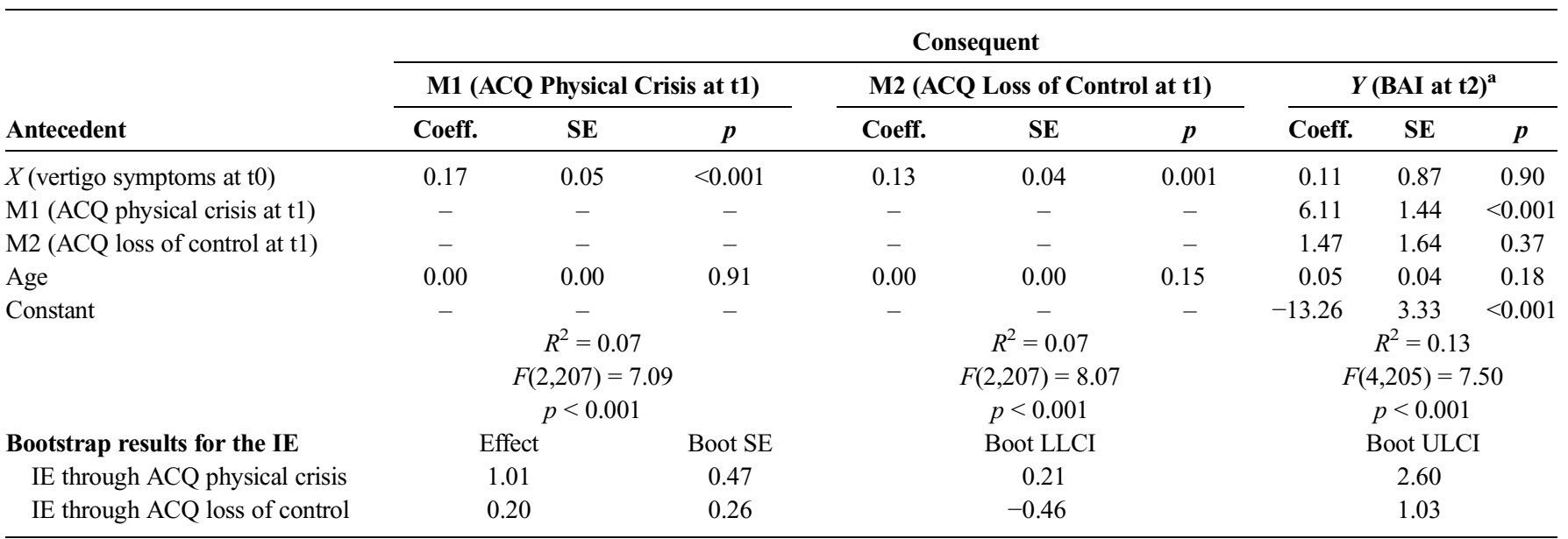

${ }^{\mathrm{a}}$ Residuals from simple linear regression analysis were entered into mediation analyses to control for BAI baseline scores.

Note: $X$ indicates independent variable; $Y$, outcome variable; M, mediator; Coeff., coefficient; SE, standard error; Boot, bootstrapping; LLCI, lower level of the $98.75 \%$ CI; ULCI, upper level of the $98.75 \%$ CI. 
secondary anxiety symptoms. This is in line with the earlier finding that persistent fear of vertigo and panic-related thoughts predicted the development of secondary panic disorder in a subgroup of VD patients with vestibular neuritis (Godemann et al., 2006); however, in our sample, only symptom-related fears remained a significant mediator when cognitions were controlled for. When taking a closer look at the ACQ subscales, only the anticipation of a physical crisis but not loss of control was a significant mediator when statistically controlling for the other subscale, even though the comparison failed to reach statistical significance.

Although the role of somatic symptom-related fears and cognitions has been well researched in functional VD and further somatoform disorders (Martin and Rief, 2011), the results at hand suggest that they come into effect irrespective of the VD symptoms' etiology. More importantly, the observation that symptom-related fears and cognitions mediate the effect VD had on psychological distress was not limited to patients with a nonorganic cause of their symptoms, but occurred across a wide range of diagnoses. Conforming to Tschan et al. (2010), high scores on the ACQ and BSQ scales were not limited to functional VD patients; in their validation study of the German Vertigo Handicap Questionnaire, those two scales did not differentiate between patients with functional versus organic VD. In line with this, Pollak et al. (2012) showed that even in an organic VD disorder with a clear understanding of its cause and straightforward treatment, benign paroxysmal positional vertigo, there appears to be a surprising rigidity of feelings and beliefs toward the illness even after treatment.

The results give rise to the question in what way dysfunctional cognitions and fears contribute to the development of psychological distress. In their cognitive behavioral model of persistent posturalperceptual dizziness, Whalley and Cane (2017) outline psychological processes in the development and maintenance of the disorder that show similarities to cognitive models of health anxiety and panic disorder. They stress the role of a) unhelpful appraisals, b) avoidance and safety behaviors, and c) attentional strategies such as selective attention to body sensations in the maintenance of dizziness that can be triggered by both organic and nonspecific VD episodes. Our results suggest that those mechanisms might come into play not only in the perpetuation of dizziness (Heinrichs et al., 2007) but also in the development of further psychopathology.

Negative perceptions and fear of somatic symptoms may lead to the avoidance of a range of activities and physical demands (Yardley, 1994a), a mechanism that has commonly been noted in panic and phobia and inspired similar treatment strategies (Edelman et al., 2012). In fact, many patients with anxiety disorders specified VD as the most anxiety-provoking and uncomfortable sensations among the bodily sensations that may be caused by panic attacks (Asmundson et al., 1998).

Although the interplay between vestibular disorders and anxiety has repeatedly been pointed out, the association with symptoms of depression seems somewhat less apparent. Nakao and Yano (2006) found that dizziness is a significant predictor of depression, with the mediating processes remaining unclear. With regard to our results, symptomrelated cognitions, loss of control in particular, might play a greater role in the development of depression compared with anxiety after VD. Patients may become passive, withdrawn, or excessively help-seeking if they feel they are not in control of their symptoms and have a low perceived self-efficacy. Indeed, in their longitudinal study with patients with recurrent vertigo, Yardley (1994b) found that handicap was negatively related to internal locus of control and positively correlated with relinquishing responsibility.

On a positive note, negative beliefs about the consequences of VD can be subject to modification in cognitive psychotherapy (Yardley et al., 2001). Dysfunctional fears and beliefs related to somatic sensations can be targeted both implicitly and explicitly: the parallels between vestibular rehabilitation and interoceptive exposure to feared sensations in cognitive behavioural therapy have been outlined before (Staab, 2011). By testing the validity of fears about VD, they not only promote psychological habituation to the symptom but can also build confidence in the predictability and controllability of symptoms. In addition, beliefs about VD can be challenged explicitly via cognitive restructuring. Providing an adequate appraisal of VD symptoms might be helpful in preventing secondary depressive and anxiety symptoms from developing and exacerbating in patients with various vestibular disorders.

Focusing on protective instead of risk factors, Tschan et al. (2011) found that patients who reported resilient coping and a higher sense of coherence were indeed less likely to develop a comorbid mental disorder 1 year after vestibular disease.

\section{Strengths and Limitations}

To our knowledge, this is the first study to investigate how symptom-related fears and beliefs contribute to the effect VD symptoms longitudinally exert on the development of anxiety and depression. The large sample includes patients with a wide range of vestibular disorders, and measurements cover a follow-up period over 1 year.

However, the study does have some methodological shortcomings. First, given the correlational and nonexperimental design of the study, no causal interpretation can be made with certainty at this stage; in light of this, results should be interpreted cautiously.

Second, the study was conducted in a tertiary care setting (i.e., a highly specialized outpatient clinic for vertigo and balance disorders) and therefore may have involved selection bias. In addition, because patients who were excluded from analyses due to missing data or loss at follow-up were significantly younger, the current sample is not fully representative for the patients' group of interest. However, the generalizability of the results might not be too strongly impaired as the age difference amounted to a small effect size only and age did not influence the results of the mediation models.

Third, the study focuses on somatic symptom-related fears and cognitions, not taking into account overall illness beliefs, that is, assumptions about the cause, course, and the expected response to treatment as well as controllability of the illness itself (not its symptoms). These illness perceptions may add to the way people cope with and respond emotionally to an illness; they have been shown to affect healthrelated quality of life and predict long-term outcome (Frostholm et al., 2007). However, we did concentrate on symptom cognitions in light of the heterogeneity of diagnoses, thereby considering that, for instance, the expectation of an illness to be long-lasting might be the result of a cognitive distortion in some patients or an unfortunately correct assumption in certain diagnostic groups such as Ménière's disease.

Fourth, it was pointed out that there is partial item overlap between the mediator and outcome measures that might have introduced bias; when analyses were repeated without respective items (specifically, items 5, 14, and 16 from the BAI), significance and effect size of the IEs did not change.

A final point of potential critique lies within the study design: measures of somatic symptom-related fears and beliefs at the first follow-up after 6 months were analyzed as mediator variables of interest. One might argue that those appraisals and fearful responses appear in closer time proximity after VD symptoms. However, the analyses yielded comparable results if baseline scores of the respective variables were entered into mediation models; the results are available from the author upon request.

\section{CONCLUSION}

Fear of bodily sensations and dysfunctional cognitions about these symptoms longitudinally contribute to the effect that initial vertigo and dizziness symptoms, irrespective of their etiology, have on the development of anxiety and depression. These findings are 
compatible with cognitive-behavioral models of psychological sequelae after VD disorders and point to the potential benefits of interventions to modify specific symptom-related beliefs and fears via different kinds of psychotherapies such as cognitive behavioural therapy.

\section{DISCLOSURE}

Parts of this project were supported by funds from the German Federal Ministry of Education and Research under grant code 01 EO 0901. The authors bear full responsibility for the content of this publication.

The authors declare no conflict of interest.

\section{REFERENCES}

American Psychiatric Association [APA] (2013) DSM- $V$. Washington, DC: APA Publishing.

Arroll M, Dancey CP, Attree EA, Smith S, James T (2012) People with symptoms of Ménière's disease: The relationship between illness intrusiveness, illness uncertainty, dizziness handicap, and depression. Otol Neurotol. 33:816-823.

Asmundson GJ, Larsen DK, Stein MB (1998) Panic disorder and vestibular disturbance: An overview of empirical findings and clinical implications. J Psychosom Res. 44: 107-120.

Barsky AJ (1992) Amplification, somatization, and the somatoform disorders. Psychosomatics. 33:28-34.

Beck AT (2005) The current state of cognitive therapy: A 40-year retrospective. Arch Gen Psychiatry. 62:953-959.

Best C, Eckhardt-Henn A, Diener G, Bense S, Breuer P, Dieterich M (2006) Interaction of somatoform and vestibular disorders. J Neurol Neurosurg Psychiatry. 77: $658-664$.

Best C, Eckhardt-Henn A, Tschan R, Dieterich M (2009a) Psychiatric morbidity and comorbidity in different vestibular vertigo syndromes. Results of a prospective longitudinal study over one year. J. Neurol. 256:58-65.

Best C, Eckhardt-Henn A, Tschan R, Dieterich M (2009b) Why do subjective vertigo and dizziness persist over one year after a vestibular vertigo syndrome? Ann NY Acad Sci. 1164:334-337.

Bigelow RT, Semenov YR, du Lac S, Hoffman HJ, Agrawal Y, Lac S (2015) Vestibular vertigo and comorbid cognitive and psychiatric impairment: The 2008 National Health Interview Survey. J Neurol Neurosurg Psychiatry. 87:367-372.

Chambless DL, Caputo GC, Bright P, Gallagher R (1984) Assessment of fear of fear in agoraphobics: The Body Sensations Questionnaire and the Agoraphobic Cognitions Questionnaire. J Consult Clin Psychol. 52:1090-1097.

Eagger S, Luxon LM, Davies RA, Coelho A, Ron MA (1992) Psychiatric morbidity in patients with peripheral vestibular disorder: A clinical and neuro-otological study. J Neurol Neurosurg Psychiatry. 55:383-387.

Eckhardt-Henn A, Best C, Bense S, Breuer P, Diener G, Tschan R, Dieterich M (2008) Psychiatric comorbidity in different organic vertigo syndromes. J Neurol. 255: $420-428$.

Edelman S, Mahoney AE, Cremer PD (2012) Cognitive behavior therapy for chronic subjective dizziness: A randomized, controlled trial. Am J Otolaryngol. 33: 395-401.

Ehlers A, Margraf J (2001) Fragebogen zu körperbezogenen Ängsten, Kognitionen und Vermeidung $(A K V)$. Göttingen: Beltz Test GmbH.

Frostholm L, Oernboel E, Christensen KS, Toft T, Olesen F, Weinman J, Fink P (2007) Do illness perceptions predict health outcomes in primary care patients? A 2-year follow-up study. J Psychosom Res. 62:129-138.

Furman JM, Balaban CD, Jacob RG, Marcus DA (2005) Migraine-anxiety related dizziness (MARD): A new disorder? J Neurol Neurosurg Psychiatry. $76: 1-8$.

Gellatly R, Beck AT (2016) Catastrophic thinking: A transdiagnostic process across psychiatric disorders. Cognit Ther Res. 40:441-452.

Godemann F, Koffroth C, Neu P, Heuser I (2004) Why does vertigo become chronic after neuropathia vestibularis? Psychosom Med. 66:783-787.
Godemann F, Schabowska A, Naetebusch B, Heinz A, Ströhle A (2006) The impact of cognitions on the development of panic and somatoform disorders: A prospective study in patients with vestibular neuritis. Psychol Med. 36:99-108.

Hautzinger M, Keller F, Kühner C (2006) Beck Depressions-Inventar Revision (BDI-II). Frankfurt am Main: Harcourt Test Services.

Hayes A (2013) Introduction to mediation, moderation, and conditional process analysis (pp 3-4). New York, NY: Guilford.

Heinrichs N, Edler C, Eskens S, Mielczarek MM, Moschner C (2007) Predicting continued dizziness after an acute peripheral vestibular disorder. Psychosom Med. 69:700-707.

Kirby SE, Yardley L (2009) Cognitions associated with anxiety in Ménière's disease. J Psychosom Res. 66:111-118.

Kühner C, Bürger C, Keller F, Hautzinger M (2007) Reliability and validity of the Revised Beck Depression Inventory (BDI-II). Results from German samples. Nervenarzt. 78:651-656.

Lahmann C, Henningsen P, Brandt T, Strupp M, Jahn K, Dieterich M, Eckhardt-Henn A, Feuerecker R, Dinkel A, Schmid G (2015) Psychiatric comorbidity and psychosocial impairment among patients with vertigo and dizziness. J Neurol Neurosurg Psychiatry. 86:302-308.

Lahmann C, Henningsen P, Dieterich M, Feuerecker R, Cyran CA, Schmid G (2012) The Munich Diagnostic and Predictor Study of Dizziness: Objectives, design, and methods. J Neurol. 259:702-711.

Limburg K, Sattel H, Radziej K, Lahmann C (2016) DSM-5 somatic symptom disorder in patients with vertigo and dizziness symptoms. J Psychosom Res. 91:26-32.

Margraf J, Ehlers A (2007) Das Beck Angst-Inventar (BAI)-Manual. Deutsche Bearbeitung. Frankfurt am Main: Harcourt Test Services GmbH.

Martin A, Rief W (2011) Relevance of cognitive and behavioral factors in medically unexplained syndromes and somatoform disorders. Psychiatr Clin North Am. 34:565-578.

Nakao M, Barsky AJ (2007) Clinical application of somatosensory amplification in psychosomatic medicine. Biopsychosoc Med. 1:17.

Nakao M, Yano E (2006) Somatic symptoms for predicting depression: One-year follow-up study in annual health examinations. Psychiatry Clin Neurosci. 60:219-225

Neuhauser HK (2007) Epidemiology of vertigo. Curr Opin Neurol. 20:40-46

Paulus MP, Stein MB (2010) Interoception in anxiety and depression. Brain Struct Funct. 214:451-463.

Pérez N, Martin E, Garcia-Tapia R (2003) Dizziness: Relating the severity of vertigo to the degree of handicap by measuring vestibular impairment. Otolaryngol Head Neck Surg. 128:372-381.

Pollak L, Segal P, Stryjer R, Stern HG (2012) Beliefs and emotional reactions in patients with benign paroxysmal positional vertigo: A longitudinal study. Am J Otolaryngol. 33:221-225.

Preacher KJ, Kelley K (2011) Effect size measures for mediation models: Quantitative strategies for communicating indirect effects. Psychol Methods. 16:93-115.

Probst T, Dinkel A, Schmid-Mühlbauer G, Radziej K, Limburg K, Pieh C, Lahmann C (2017) Psychological distress longitudinally mediates the effect of vertigo symptoms on vertigo-related handicap. J Psychosom Res. 93:62-68.

Schmid G, Henningsen P, Dieterich M, Sattel H, Lahmann C (2011) Psychotherapy in dizziness: A systematic review. J Neurol Neurosurg Psychiatry. 82:601-606.

Staab JP (2011) Behavioral aspects of vestibular rehabilitation. NeuroRehabilitation. 29:179-183.

Staab JP, Ruckenstein MJ (2003) Which comes first? Psychogenic dizziness versus otogenic anxiety. Laryngoscope. 113:1714-1718

Tschan R, Best C, Beutel ME, Knebel A, Wiltink J, Dieterich M, Eckhardt-Henn A (2011) Patients' psychological well-being and resilient coping protect from secondary somatoform vertigo and dizziness (SVD) 1 year after vestibular disease. J Neurol. 258:104-112.

Tschan R, Wiltink J, Best C, Bense S, Dieterich M, Beutel ME, Eckhardt-Henn A (2008) Validation of the German version of the Vertigo Symptom Scale (VSS) 
in patients with organic or somatoform dizziness and healthy controls. J Neurol. 255:1168-1175.

Tschan R, Wiltink J, Best C, Beutel M, Dieterich M, Eckhardt-Henn A (2010) Validation of the German version of the Vertigo Handicap Questionnaire (VHQ) in patients with vestibular vertigo syndromes or somatoform vertigo and dizziness. Psychother Psychosom Med Psychol. 60:e1-e12.

Tusa RJ (2009) Dizziness. Med Clin North Am. 93:263-271.

Vîslă A, Flückiger C, Grosse Holtforth M, David D (2016) Irrational beliefs and psychological distress: A meta-analysis. Psychother Psychosom. 85:8-15.

Weidt S, Bruehl AB, Straumann D, Hegemann SC, Krautstrunk G, Rufer M (2014) Health-related quality of life and emotional distress in patients with dizziness: A cross-sectional approach to disentangle their relationship. BMC Health Serv Res. 14:317.

Whalley MG, Cane DA (2017) A cognitive-behavioral model of persistent posturalperceptual dizziness. Cogn Behav Pract. 24:72-89.
Wiltink J, Tschan R, Michal M, Subic-Wrana C, Eckhardt-Henn A, Dieterich M, Beutel MA (2009) Dizziness: Anxiety, health care utilization and health behaviorresults from a representative German community survey. $J$ Psychosom Res. $66: 417-424$.

Yardley L (1994a) Contribution of symptoms and beliefs to handicap in people with vertigo: A longitudinal study. Br J Clin Psychol. 33:101-113.

Yardley L (1994b) Prediction of handicap and emotional distress in patients with recurrent vertigo: Symptoms, coping strategies, control beliefs and reciprocal causation. Soc Sci Med. 39:573-581.

Yardley L, Beech S, Weinman J (2001) Influence of beliefs about the consequences of dizziness on handicap in people with dizziness, and the effect of therapy on beliefs. J Psychosom Res. 50:1-6.

Yardley L, Masson E, Verschuur C, Haacke N, Luxon L (1992) Symptoms, anxiety and handicap in dizzy patients: Development of the Vertigo Symptom Scale. J Psychosom Res. 36:731-741. 